\title{
Psychosocial issues associated with stroke
}

\section{Opinion}

A stroke is a sudden and often traumatic major life event and often results in life-changing consequences with which affected people must cope. Stroke is a common cause of disability in adults and more than one-third of people who survive a stroke will have severe disability. ${ }^{1}$ A person may never look at themselves or their life in the same manner. The person may no longer be able to do many of the things that they felt defined them, gave their life meaning, or brought them enjoyment. The initial post-stroke focus is often on physical impairments such as hemiplegia or language problems such as aphasia, but over time the stress and burden of the situation often takes a toll on the individual's mood and caregivers.

\section{Toll of psychosocial adjustment issues}

Between $35 \%$ and $75 \%$ of patients will have significant cognitive impairment, ${ }^{2,3}$ about one-third of people suffer depression after stroke, ${ }^{4}$ and about one-fourth suffer significant anxiety, ${ }^{5}$ and about $20 \%$ suffer from insomnia. ${ }^{6}$ These psychological issues have been shown to negatively influence rehabilitation and outcomes. For example, post-stroke depression has been shown to be related to more negative functional outcomes. ${ }^{7,8}$ The psychological issues may affect rehabilitation outcomes through a reduction in follow-through with practice and home exercise program, reduced energy level, increase fatigue, reduced frustration tolerance and potentially less motivation and hope about the future. For example, depression can cause many of the aforementioned symptoms, yet these symptoms may be mistaken for medical or personality issues. Depression is a significant complication of stroke that may impede rehabilitation, recovery, quality of life, and caregiver health. ${ }^{9-11}$ Furthermore, stroke-associated depression may reduce survival and increase the risks of recurrent vascular events. ${ }^{12,13}$ Despite the vast manner in which psychological issues can affect the person with a stroke, much of the literature on the topic suggests that people often do not receive psychological treatment after their stroke. Beyond the psychological disorders that can occur, there are a vast number of emotional issues that can affect the person and their caregiver. Some of these issues may involve limited awareness or insight into impairments and the potential effects of these issues on safety and participation in rehabilitation. Because of the constellation of symptoms associated with stroke, it is often helpful to have psychologists, neuropsychologists, and social workers involved in stroke rehabilitation. Several organizations have suggested that inclusion of these disciplines in stroke care should be the standard. The evidence-based National Institute for Health and Care Excellence (NICE) quality standards exist for stroke ${ }^{14}$ and suggest the psychology is a core component of appropriate stroke care.

Some of the research on stroke outcomes suggests that patients treated by stroke-specialists, and others with relevant stroke-specific knowledge and skills, are more likely to survive their stroke, to return home, and to become independent. ${ }^{15,16}$ In terms of specific techniques to help with mood, cognitive behavioral therapy has been shown to be effective with this population. ${ }^{4}$ Hackett $\&$ colleagues ${ }^{4}$ found a weak role for antidepressant medication post stroke. They questioned if "pharmacotherapy is effective in either producing a remission or
Volume 9 Issue 5 - 2018

Robert Perna, Jessica Le MS

The Institute of Rehabilitation Research, Texas, USA

Correspondence: Robert Perna, The Institute of Rehabilitation Research, Houston, Texas, USA, Email dr.parna@juno.com

Received: July 10, 2017 | Published: October 15, 2018

in preventing the onset of diagnosable depressive illness in stroke patients." They concluded, by saying "it is uncertain how clinically significant such modest changes in mood scores are for stroke patients who would be considered to have depressive symptoms within the mild- moderate range." These findings are consistent with metaanalyses suggesting a small treatment effect size for antidepressants.

\section{Conclusion and recommendations}

Stroke is a complex disorder that not only commonly affects physical and cognitive functioning, but also often affects mood and diverse psychosocial variables. Understanding how it affects people is essential to effectively conceptualizing patient needs and providing the appropriate care. Appropriate and efficacious stroke care requires good interdisciplinary communication and the involvement of clinicians who are experienced in the understanding of stroke symptoms and sequelae. Given the high incidence of comorbid emotional issues, it is essential that those issues are evaluated and treated. Stroke care should include screening for emotional adjustment issues and evidence-based treatment (such as cognitive behavior therapy and/or antidepressant medication) for depression and other diagnosable disorders.

\section{Acknowledgements}

None.

\section{Conflict of interest}

The author declares that there is no conflict of interest.

\section{References}

1. Mayo N E, Wood-Dauphinee S, Ahmed S Anon. Tackling the global burden of stroke. Lancet Neurology. 2005;4:689.

2. Tatemichi TK, Desmond DW, Stern Y, et al. Cognitive impairment after stroke: frequency, patterns and relationship to functional abilities. $J$ Neurol Neurosurg Psychiatry. 1994;57(2):202-207.

3. Nys GM, van Zandvoort MJ, de Kort PL, et al. Cognitive disorders in acute stroke: Prevalence and clinical determinants. Cerebrovasc Dis. 2007;23(5-6):408-416.

4. Hackett, Yapa C, Parag V, et al. Frequency of depression after stroke: A systematic review of observational studies. Stroke. 2005;36(6):1330-1340.

5. Barker-Collo S. Depression and anxiety three months post-stroke: Prevalence and correlates. Arch Clin Neuropsychol. 2007;22(4):519-531.

6. Leppävuoria, Pohjasvaara T, Vataja R, et al. Insomnia in Ischemic Stroke Patients. Cerebrovascular Disease. 2002;14(2):90-97. 
7. Kneebone I, Dunmore E. Psychological management of post-stroke depression. Br J Clin Psychol. 2000;39(Pt 1):53-66.

8. Matsuzaki S, Hashimoto M, Yuki S, et al. The relationship between poststroke depression and physical recovery. JAffect Disord. 2015;176:56-60.

9. Sinyor D, Amato P, Kaloupek DG, et al. Post-stroke depression: relationships to functional impairment, coping strategies, and rehabilitation outcome. Stroke. 1986;17(6):1102-1107.

10. Robinson RG, Bolla-Wilson K, Kaplan E, et al. Depression influences intellectual impairment in stroke patients. Br J Psychiatry. 1986;148:541547.

11. Anderson CS, Linto J, Stewart-Wynne EG. A population-based assessment of the impact and burden of caregiving for long-term stroke survivors. Stroke. 1995;26(5):843-849.
12. House A, Knapp P, Bamford J, et al. Mortality at 12 and 24 months after stroke may be associated with depressive symptoms at 1 month. Stroke. 2001;32(3):696-701

13. Morris PL, Robinson RG, Andrzejewski P, et al. Association of depression with 10-year poststroke mortality. Am J Psychiatry. 1993;150(1):124 129.

14. The National Institute for Health and Clinical Excellence Quality Standard for Stroke (2010).

15. National Audit Office, Reducing Brain Damage: Faster access to better stroke care, Norwich: Stationery Office, 2005.

16. Cochrane Database of Systematic Reviews. UK Forum for Stroke Training; 2007. 